\title{
Novel gratings for next-generation instruments of astronomical observations
}

N. Ebizuka, T. Okamoto, M. Takeda, T. Hosobata, Y. Yamagata, et al.

N. Ebizuka, T. Okamoto, M. Takeda, T. Hosobata, Y. Yamagata, M. Sasaki, M. Uomoto, T. Shimatsu, S. Sato, N. Hashimoto, I. Tanaka, T. Hattori, S. Ozaki, W. Aoki, "Novel gratings for next-generation instruments of astronomical observations," Proc. SPIE 10233, Holography: Advances and Modern Trends V, 102330M (19 June 2017); doi: 10.1117/12.2264892

SPIE. Event: SPIE Optics + Optoelectronics, 2017, Prague, Czech Republic 


\title{
Novel gratings for next-generation instruments of astronomical observations
}

\author{
N. Ebizuka*a , T. Okamoto ${ }^{\mathrm{a}}$, M. Takeda ${ }^{\mathrm{a}}$, T. Hosobata ${ }^{\mathrm{a}}$, Y. Yamagata $^{\mathrm{a}}$, M. Sasaki ${ }^{\mathrm{b}}$, M. Uomoto $^{\mathrm{c}}$, \\ T. Shimatsu ${ }^{\text {c }}$ S. Sato, N. Hashimoto ${ }^{\text {d }}$ I. Tanaka ${ }^{\mathrm{e}}$, T. Hattori ${ }^{\mathrm{e}}$, S. Ozaki ${ }^{\mathrm{e}}$, W. Aoki ${ }^{\mathrm{e}}$ \\ ${ }^{a}$ RIKEN, 2-1 Hirosawa, Wako, Saitama, Japan 351-0198, ${ }^{\mathrm{b}}$ Faculty of Engineering, \\ Toyota Technological Institute, ${ }^{c}$ Frontier Research Institute for Interdisciplinary Sciences, \\ Tohoku University, ${ }^{\mathrm{d}}$ CITIZEN WATCH Co. Ltd., ${ }^{\mathrm{e}}$ National Astronomical Observatory of Japan
}

\begin{abstract}
We will introduce current status of development of a birefringence volume phase holographic (B-VPH) grating, volume binary (VB) grating and reflector facet transmission (RFT) grating developing as the novel dispersive optical element for astronomical instruments for the $8.2 \mathrm{~m}$ Subaru Telescope, for next generation $30 \mathrm{~m}$ class huge ground-based telescopes and for next generation large space-bone telescopes. We will also introduce a hybrid grism developed for MOIRCS (Multi-Object InfraRed Camera and Spectrograph) of the Subaru Telescope and a quasi-Bragg (QB) immersion grating. Test fabrication of B-VPH gratings with a liquid crystal (LC) of UV curable and normal LCs or a resin of visible light curable are performed. We successfully fabricated VB gratings of silicon as a mold with ridges of a high aspect ratio by means of the cycle etching process, oxidation and removal of silicon oxide. The RFT grating which is a surface-relief (SR) transmission grating with sawtooth shaped ridges of an acute vertex angle. The hybrid grism, as a prototype of the RFT grating, combines a high-index prism and SR transmission grating with sawtooth shape ridges of an acute vertex angle. The mold of the SR grating for the hybrid grism on to a work of Ni-P alloy of non-electrolysic plating successfully fabricated by using our ultra-precision machine and a single-crystal diamond bite. The QB immersion grating was fabricated by a combination of an inclined QB grating, Littrow prism and surface reflection mirror.
\end{abstract}

Keywords: Volume grating, Echelle spectroscopy, RCWA

\section{INTRODUCTION}

As the diameter of the primary mirror of an astronomical telescope becomes larger, the light-gathering power is increased, with the result that the astronomical objects and its scope of application of the astronomical spectrograph are expanded. For example, in exploration and research for extra-solar planets, which has made rapid progress in recent years, we can detect Earth's mass planets from observations of radial (line of sight) velocity variations of the central star using a high dispersion spectrograph, and it is becoming possible to analyze the components of a planetary atmosphere by spectroscopic observation when a planet transits in front of the central star. However, in the case of a ground-based telescope without adaptive optics, the image size of a star on the sky is determined by the atmospheric turbulence (typically, about 1 arcsecond), so when the aperture of a telescope becomes large, the image size of a star also increases on the focal plane. As the slit width is proportional to the image size of the star, the size of an astronomical spectrograph has to be increased proportional to the aperture size of the telescope (the volume is the cube of the aperture) in order to achieve the same resolving power at the same wavelength. On the other hand, in the case of a space-bone telescope, since the diffraction limited observations can be achieved, even if the aperture of the telescope is changed, the size of the astronomical spectrograph that the wavelength and the resolving power are the same, does not change. However, the limitations on the weight and the volume for the space equipment are extremely strict. As a result, large angular dispersion and high efficiency are required for a dispersive optical element for a next generation large telescope in order to reduce the size and the weight of an astronomical spectrograph. Furthermore, as number of pixels of a visible and infrared imaging detector has become large, the echelle spectroscopy which combines a higher order diffraction grating with large angular dispersion and a prism or a diffraction grating of the first diffraction order as a vertical dispersive element, is widely used in order to observe a wide wavelength range simultaneously.

Therefore, the developments of transmission gratings with large angular dispersion are required since a collimator and imaging optical element are able to place nearby the transmission grating, and it can be reduced the size of a spectrograph. Various kinds of surface-relief (SR) transmission gratings (Fig. 1) and volume phase holographic (VPH) gratings (Fig. 2), especially grisms (direct vision grating), are developed for astronomical instruments of the $8.2 \mathrm{~m}$

Holography: Advances and Modern Trends V, edited by Miroslav Hrabovský, John T. Sheridan, Antonio Fimia, Proc. of SPIE Vol. 10233, 102330M - @ 2017 SPIE · CCC code: 0277-786X/17/\$18 · doi: 10.1117/12.2264892 

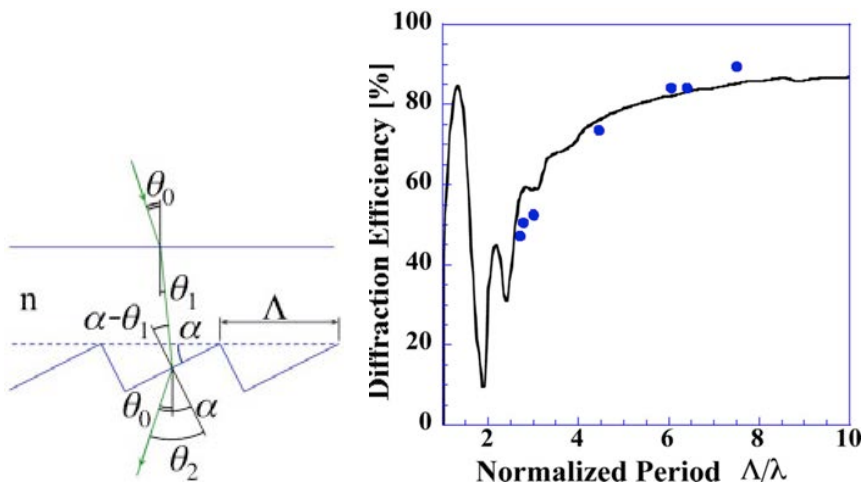

Figure 1 Propagation of incident beam in SR transmission grating with sawtooth shape ridges (left) and diffraction efficiency versus lattice period normalized by peek wavelength (right) $[2,7,8]$.
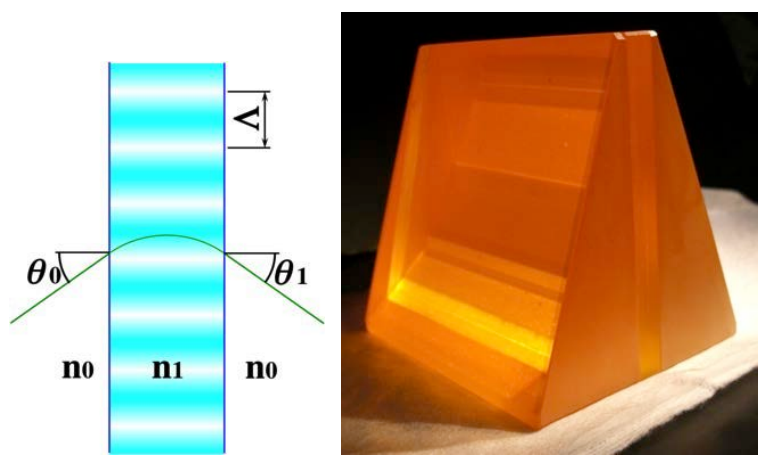

Figure 2 Schematic representation of VPH grating (left) and VPH grism with high refraction index (ZnSe) prism (right).

Subaru telescope [1-4] and domestic and foreign telescopes [5, 6]. The SR transmission grating with sawtooth shape grooves (Fig. 1 left) has to increase the refractive index of ridges according as the angular dispersion increases [2,7]. For example, when the incident and the diffraction angles are $45^{\circ}$, since a refractive index of ridges of a SR transmission grating has to be 2.3 or more, it is not possible to use an optical glass or resin. Furthermore, when the lattice period is less than four times of the wavelength, the diffraction efficiency of the first diffraction order of a SR transmission grating steeply decreases (Fig. 1 right) [8].

The VPH grating is a thick diffraction grating in which the refractive index is modulated sinusoidally (Fig. 2 left), and when the lattice period is 0.7 to 4 times of the wavelength, the diffraction efficiency can be achieved up to $100 \%$ for $S$ or $P$ polarization of the 1st diffraction order [8-10]. The various VPH gratings and VPH grisms (Fig. 2 right) are mounted in many astronomical instruments in order to achieve large angular dispersion compared with a SR transmission grating of a same effective aperture size. However diffraction efficiency of a VPH grating decreases for a natural polarization and circular polarization according as a diffraction angle increases because the properties of the diffraction efficiency become different between $S$ and $P$ polarizations as shown in figure 3. Moreover, a VPH grating is not suitable for an echelle spectrograph because diffraction efficiency of the VPH grating decreases as the diffraction order increases.

We will introduce a birefringence VPH $(\mathrm{B}-\mathrm{VPH})$ grating $[2,7,11]$, the volume binary (VB) grating [12, 13] and reflector facet transmission (RFT) grating [7] developing as the novel dispersive optical elements for astronomical instruments for the Subaru Telescope, for the TMT (Thirty Meter Telescope) [14], and for next generation instruments of ground-based telescopes [15, 16] and space-bone telescopes [17]. We will also introduce a hybrid grism [7] developed for MOIRCS (Multi-Object InfraRed Camera and Spectrograph) [18] of the Subaru Telescope and a quasi-Bragg (QB) immersion grating [11]. The hybrid grism is combined a high-index prism with a replicated SR transmission grating of acute ridges angle. The immersion grating has the big advantage for an astronomical spectrograph especially in the infrared wavelength since the size of a spectrograph can be dramatically reduced by using an immersion grating of silicon $(\mathrm{n} \sim 3.3)$ or germanium $(\mathrm{n} \sim 4.0)$ or a material with a high-refractive index.
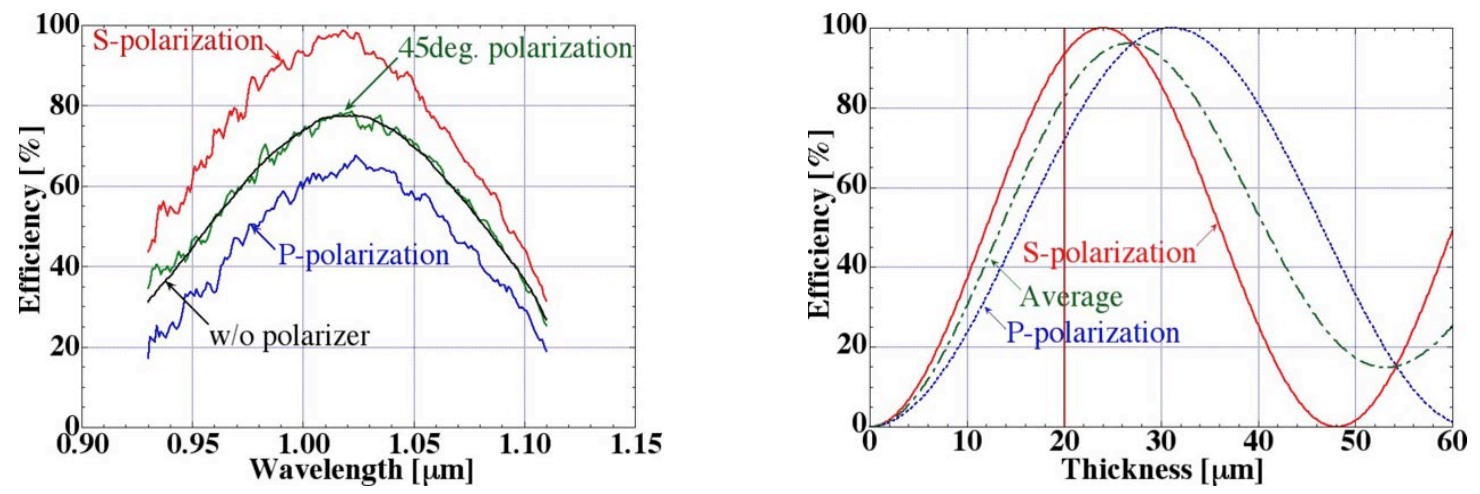

Figure 3 Measured polarized diffraction efficiency (left) and calculated polarized diffraction efficiency versus grating thickness of MOIRCS VPH grating (right), $\mathrm{n}_{\max }=1.547, \mathrm{n}_{\min }=1.513, \Lambda=0.984 \mu \mathrm{m}$ and $\theta_{\mathrm{B}}=19.8^{\circ}$ at $\lambda=1.02 \mu \mathrm{m}[3,11]$. 

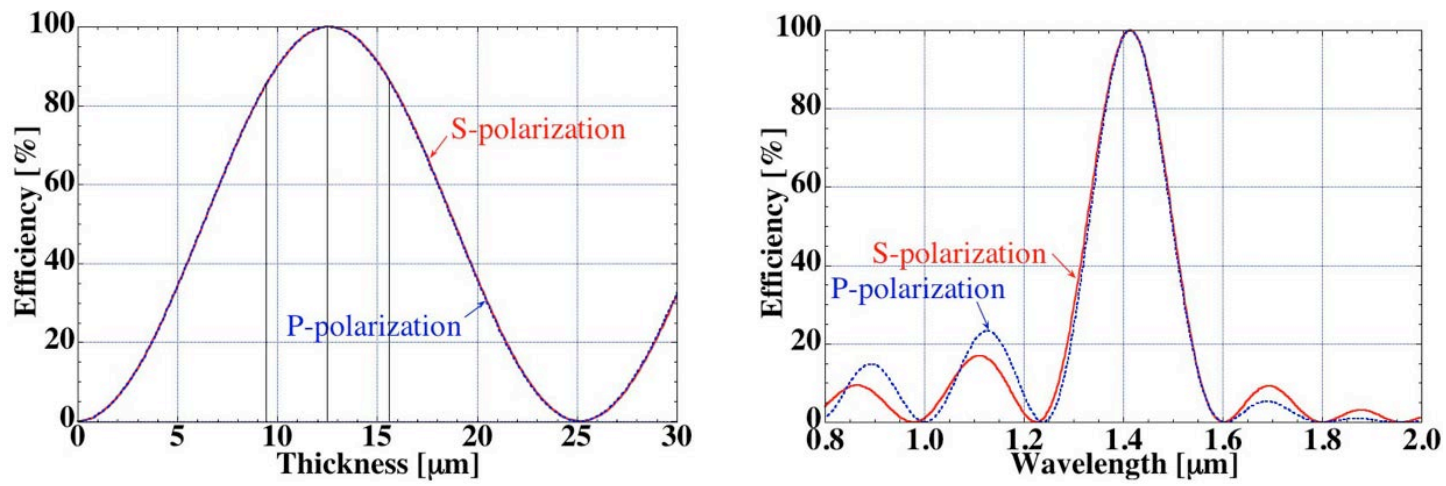

Figure 4 Calculated polarized diffraction efficiencies versus grating thickness $t$ of birefringence VPH grating at $\lambda$ $=1.41 \mu \mathrm{m}$ (upper panel), and polarized spectral diffraction efficiencies on $\mathrm{t}=12.5 \mu \mathrm{m}$ (left). $n_{1}=1.55, n_{\mathrm{S} \max }=1.60$, $n_{\mathrm{S} \min }=1.50, n_{\mathrm{P} \max }=1.653, n_{\mathrm{P} \min }=1.50, \Lambda=1.0 \mu \mathrm{m}, \theta_{0}=45^{\circ}$ (right) $[2,7]$.

\section{NOVEL GRATINGS}

\subsection{Birefringence VPH grating}

In the VPH grating, when an optically anisotropic medium such as liquid crystal (LC) and an isotropic medium or two kinds of optically anisotropic media are combined as a hologram recording material, the refractive index of an optically anisotropic medium and isotropic medium or two kinds of optically anisotropic media are adjusted so that the diffraction efficiency characteristics of the $S$ and $P$ polarizations at the first diffraction order coincide with each other, it is possible to achieve high diffraction efficiency even for a natural polarization and circular polarization. Figure 4 shows a numerical calculation of the diffraction efficiency of a birefringence VPH grating by using our own software of the rigorous coupled-wave analysis (RCWA) method [19, 20] modified for an anisotropic medium [2, 7].

We mixed an ultraviolet (UV) curable LC (no $=1.55$, ne $=1.72)$ and normal LC (no=1.5, ne $=1.65)$, sandwiched between two glass substrates in CITIZEN WATCH Co. Ltd., and the B-VPH grating was prototyped by an exposure optical system of a two-beams interferometer with an ultraviolet $(325 \mathrm{~nm})$ laser. As shown on the left in figure 5, this B-VPH grating has diffraction efficiencies of up to $66 \%, 27 \%$ and $42 \%$ for $\mathrm{S}, \mathrm{P}$ and natural polarizations, respectively. In addition, a visible light curable resin $(\mathrm{n}=1.51)$ and the UV-curable $\mathrm{LC}(\mathrm{no}=1.55$, ne $=1.72)$ were mixed and sandwiched between two glass substrates, and the B-VPH grating was prototyped by an exposure optical system of a two-beams interferometer with a visible $(532 \mathrm{~nm})$ laser. As shown on the right in figure 5, this B-VPH grating achieves diffraction efficiencies of up to $80 \%$ and $86 \%$ for $S$ and $P$ polarizations, respectively.
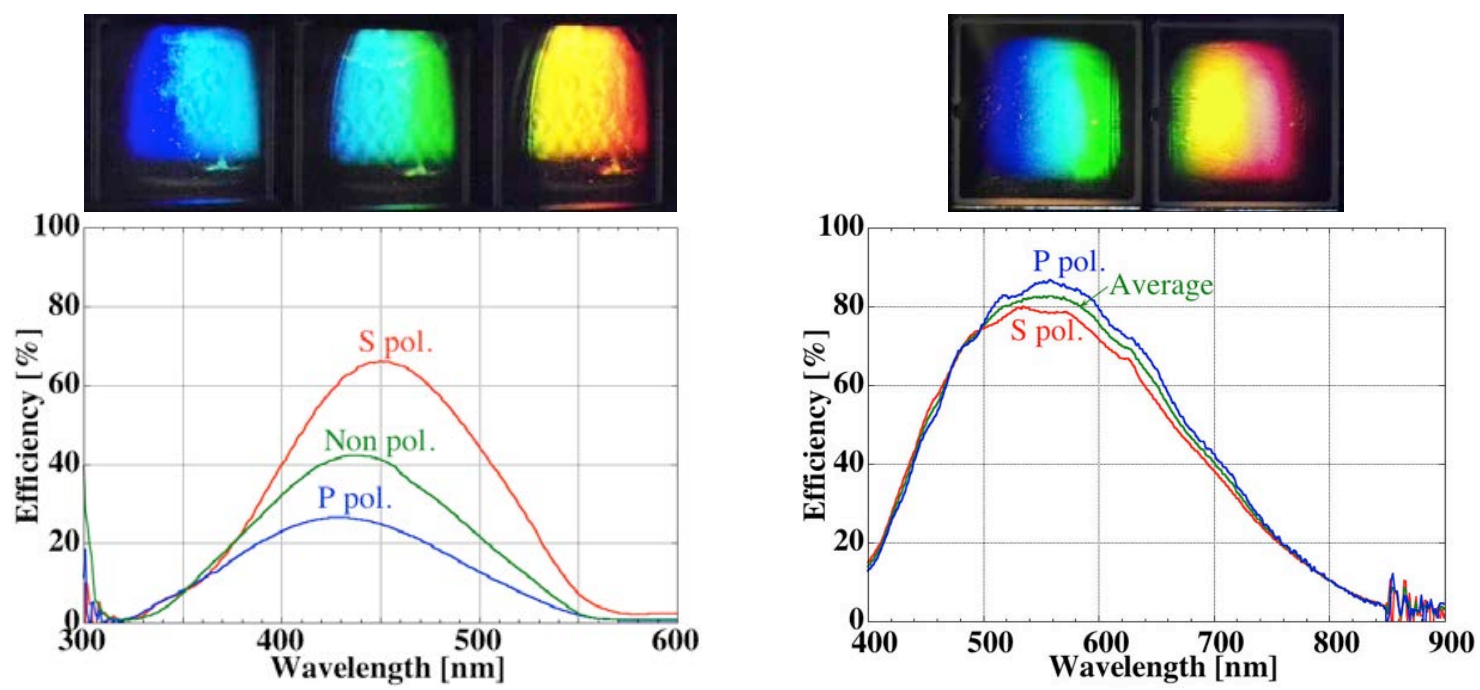

Figure 5 Diffraction efficiency of B-VPH grating. Combination of UV-curable LC (no=1.55, ne=1.72) and normal LC $(\mathrm{no}=1.5, \mathrm{ne}=1.65), \Lambda \sim 0.45 \mu \mathrm{m}(\mathrm{t}=10 \mu \mathrm{m}, 2,240 \mathrm{groove} / \mathrm{mm}), \mathrm{t}=10 \mu \mathrm{m}$ (left), and combination of visible-curable resin $(\mathrm{n}=1.51)$ and $\mathrm{UV}$-curable LC (no=1.55, $\mathrm{ne}=1.72), \Lambda \sim 1.6 \mu \mathrm{m}(616$ groove $/ \mathrm{mm}), \mathrm{t}=20 \mu \mathrm{m}$ (right) 

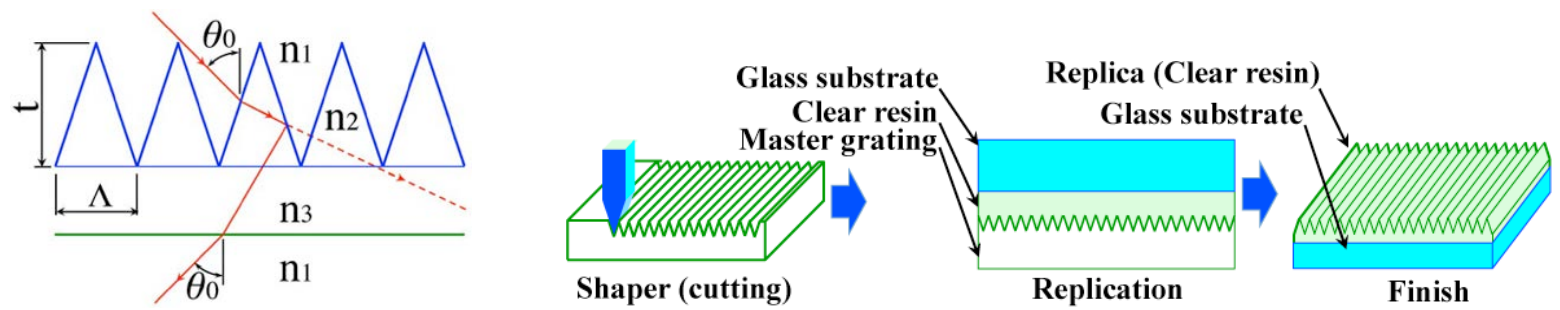

Figure 6 Schematic representation of RFT grating (left), and fabrication process of SR transmission grating with ridges of acute angle (right) [7].

\subsection{Reflector facet transmission grating and hybrid grism}

The RFT grating is a SR transmission grating with a new lattice structure of sawtooth shaped ridges with an acute vertex angle in which an incident beam from one facet of a ridge is reflected on the other facet of the ridge and the beam is exited from the rear surface of the ridge as shown on the left in figure 6 [7]. In the case of the incident and the diffraction angle are $45^{\circ}$, and the refractive index of ridges is $\mathrm{n}=1.54$, the vertex angle of the ridges is $38.8^{\circ}$. It was found that the RFT grating is able to achieve diffraction efficiency of above $80 \%$ from the 4th to higher diffraction orders by numerical calculations using the RCWA method [7]. We had planed to prototype a RFT grating for the WFOS (Wide Field Optical Spectrograph) [21] of the TMT by a replica from a mold in which V grooves were processed on a work of nickel-phosphorus alloy by shaper cutting with our ultra-high precision machine and a single-crystal diamond tool (Fig. 6 right). Since ridges of RTF gratings for the WFOS have the small vertex angles from $35^{\circ}$ to $44^{\circ}$, difficulties are expected in the process of the mold machining and the replication. So we decided to prototype a SR transmission grating for a hybrid grism of the MOIRCS to clarify the problem in fabrication of the RTF grating for the WFOS. The grating period of the SR transmission grating is $10.8 \mu \mathrm{m}$ and the vertex angle is about $60^{\circ}$.

The medium dispersion (R1300) grism for MOIRCS with saw tooth ridges is directly machined onto a prism of KRS-5 (mixed crystal of thallium chloride and thallium bromide) by a ruling engine. However, fine cracks have seen on the surface and the efficiency is remarkably deteriorated because this grism was repeated heat cycle between room temperature and $100 \mathrm{~K}$ when the enclosure of the MOIRCS was opened and closed. So we decided to develop a hybrid grism combining SR transmission grating of replica $(\mathrm{n} \sim 1.5)$ with acute ridge angle and $\mathrm{ZnSe}(\mathrm{n}=2.45 @ 1.65 \mu \mathrm{m})$ prism (Fig. 7 left). The mold of a surface relief grating for the hybrid grism was successfully fabricated on to a work of Ni-P alloy by shaper cutting with our ultra-precision machine and a single-crystal diamond bite (Fig 7 middle and right).

Then, experiments of replication were made 5 times by a company of nanoimprint manufactures. Until the 1 st to the 4th experiments UV curable resin was used, however it was not practical because peelings and large wavefront error of transmission due to the shrinkage at the curing. In the 5th experiment, a two-part resin with small curing shrinkage mixed with silica was used, however the most of replica peeled off from the glass substrate, despite the use of a coupling agent. The replication process is currently being improved.

The peak diffraction efficiencies of the first replica prototype were about $45 \%$ (Fig. 8 left). Numerical calculations of the spectral characteristics for this SR transmission grating by the RCWA method revealed that the diffraction efficiency was lowered because the wavelength range shifted to the shorter wavelength (Fig. 8 left). Therefore, we investigated the spectral characteristics of the diffraction efficiency by changing the angle of the facet $(\beta)$ from which the beam exits and the vertex angle $(\gamma)$ of the ridge. As a result, in the case of the lattice shape $\beta=65.5^{\circ}$ and $\gamma=55.0^{\circ}$, it was found that the wavelength range and the diffraction efficiency are optimal (Fig. 8 right).
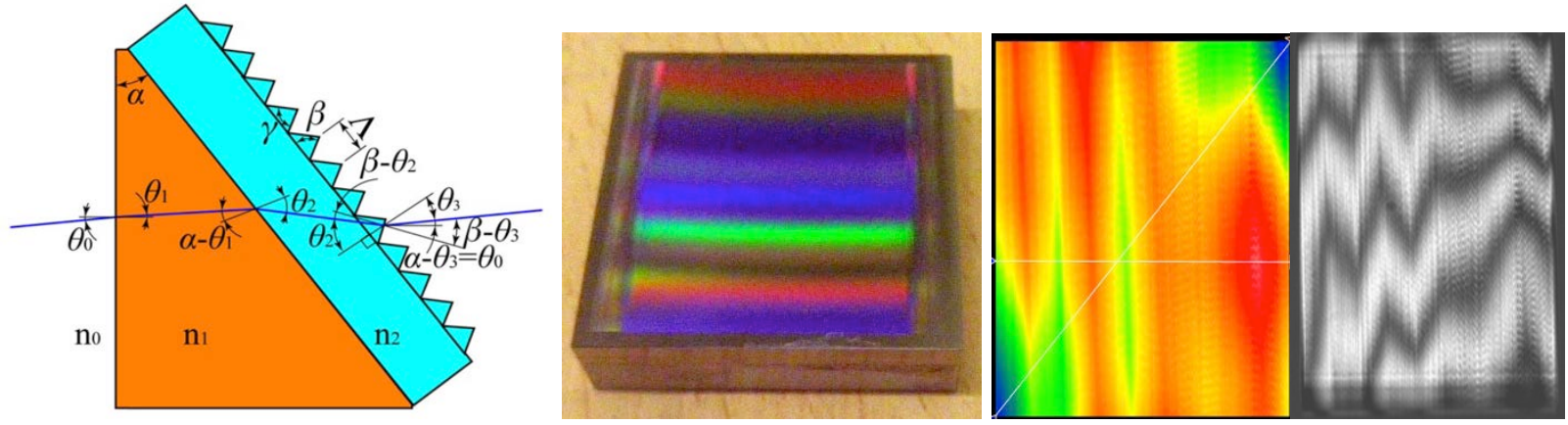

Figure 7 Schematic representation of hybrid grism [7] (left), mold for replication experiment with work piece size of $50 \times 50$ [mm] (middle), wave-front error and interferogram of mold (right). 

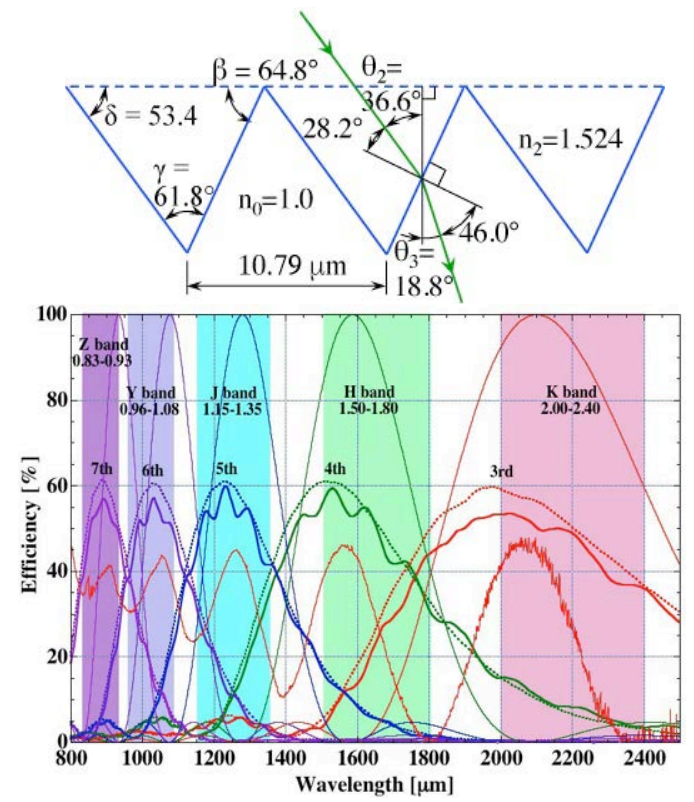

Fig. 8 Diffraction efficiency of MOIRCS hybrid grism. Left: current design, $\beta=64.8^{\circ}, \gamma=61.8^{\circ}$. Right: optimal design, $\beta=65.5^{\circ}, \gamma=55.0^{\circ}$.

\subsection{Quasi-Bragg grating and volume binary grating}

Quasi-Bragg (QB) grating is a transmission grating with a new structure in which rectangular metal films or low refractive index layers as mirror surfaces are aligned in parallel at an equal interval like a window shade or an armor window $[2,7,11]$. It is confirmed by numerical calculations using the RCWA method, that a QB grating can achieve high diffraction efficiency up to $80 \%$ in higher diffraction orders [22]. As in a VPH grism, a QB grism as shown on the left in figure 9 has a structure in which a QB grating is sandwiched between two prisms, so that the limitation of the critical angle at the interface between the high-index prism and the QB grating is less strict compared with a hybrid grism (Fig. 7 left), it is suitable for a high dispersion spectroscopy and becomes a promising dispersing optical element for an astronomical imaging instrument if it is put into practical use.

We decided to develop a QB grism for the high dispersion grism of nu-MOIRCS. The nu-MOIRCS is scheduled as the first light instrument for the ULTIMATE Subaru. The ULTIMATE Subaru plans to install a new ground-layer adaptive optics (GLAO) onto the Subaru telescope, improve the limiting magnitude and the spatial resolution by reducing the size of the star image by correcting the wavefront error due to the atmospheric turbulences. The echelle grism for the spectra from the third to the sixth diffraction orders combined with a direct vision prism as a cross disperser installs in the nu-MOIRCS, it can simultaneously covert the wavelength range of 0.8 to $1.8 \mu \mathrm{m}$ with large angular dispersion by folding the spectra on the detector, and it is the key to improve performance of the nu-MOIRCS.

By numerical calculations using the RCWA method, a QB grating uses the total reflection by the medium of a low
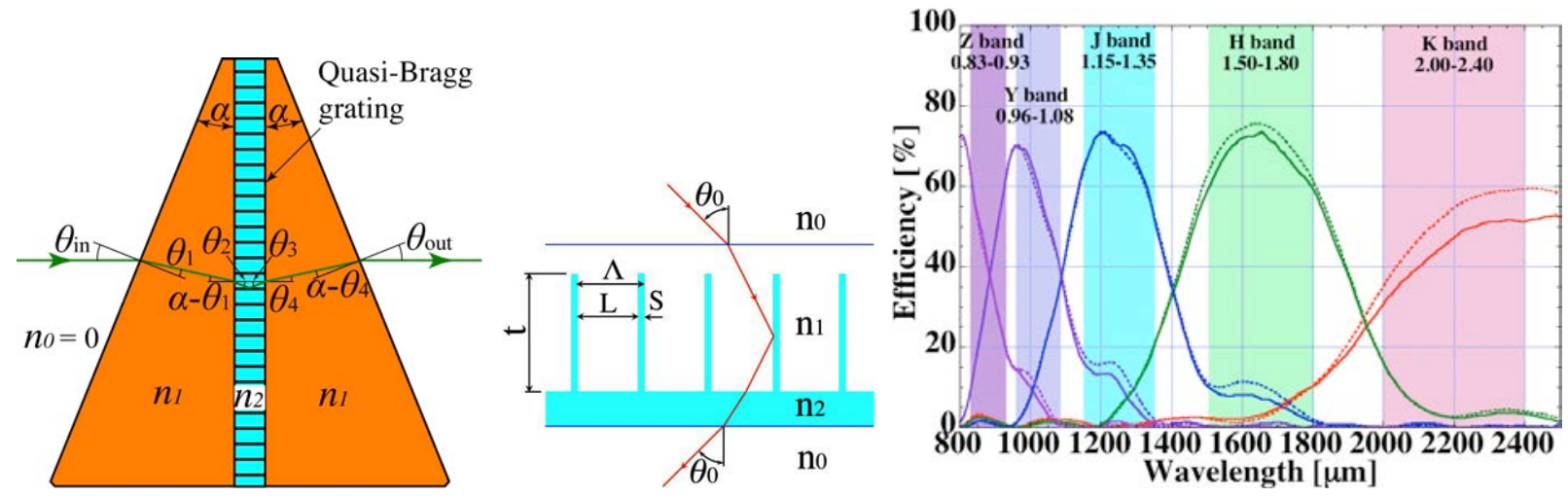

Figure 9 Schematic representation of QB grism (left) and VB grating (center), diffraction efficiency of VB grating for echelle grism of nu-MOIRCS (right: $\theta_{0}=28.4^{\circ}, \mathrm{n}_{1}=1.33, \mathrm{n}_{2}=\mathrm{n}_{3}=1.6, \Lambda=5.1 \mu \mathrm{m}, \mathrm{L} \& \mathrm{~S}=4.6: 0.5[\mu \mathrm{m}], \mathrm{t}=16 \mu \mathrm{m}$ ). 

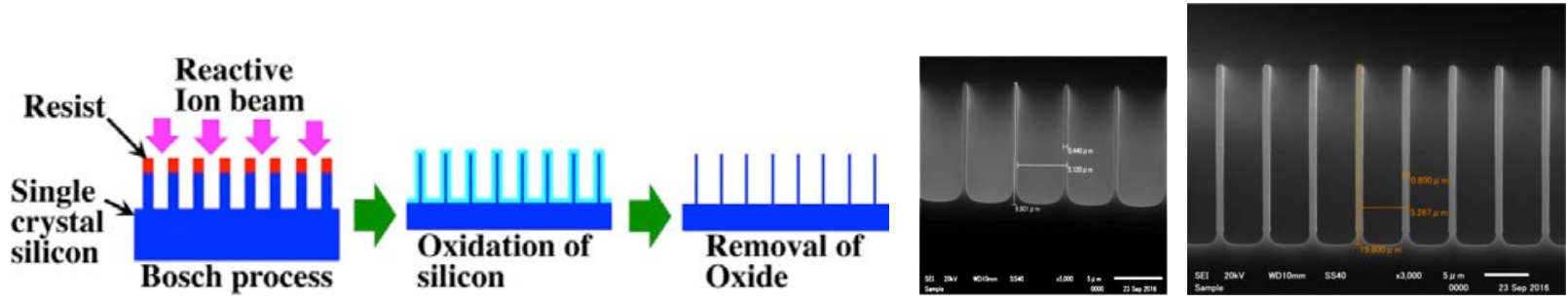

Figure 10 Fabrication process for silicon VB grating (left to middle) as a mold, central right: $\Lambda=5.1 \mu \mathrm{m}, \mathrm{t}=10 \mu \mathrm{m}, \mathrm{s}=0.44 \mu \mathrm{m}$, most right: $\Lambda=5.1 \mu \mathrm{m}, \mathrm{t}=20 \mu \mathrm{m}, \mathrm{s}=0.80 \mu \mathrm{m}$.

refractive index as the mirror surface as shown on the center in figure 9, that is, a VB grating with a high aspect ratio functioning as a QB grating, we found that high diffraction efficiencies can be achieved by the grating [2, 7, 23]. We decided to develop a VB grating for the echelle grism of the nu-MOIRCS. On the right in figure 9 shows the diffraction efficiency of the VB grating (lattice period $\Lambda=5.1 \mu \mathrm{m}, \mathrm{L} \& \mathrm{~S}=4.6: 0.5[\mu \mathrm{m}]$, thickness $\mathrm{t}=16 \mu \mathrm{m}$ ) for the echelle grism. The echelle grism is fabricated by sandwiching the VB grating between two ZnSe prisms.

We develop a fabrication method for a VB grating as a mold of silicon with ridges of high aspect ratio in Toyoda Institute of Technology. By numerous trial fabrications, we found the process which oxygen is slightly added to the etching gas and the passivation gas, etching and sidewall protection (Bosch process) are performed while cleaning, and the amount of an etching volume is reduced. As a result, the scallops (irregularities formed every etching cycle) on the sidewall of the ridges successfully reduced to about $40 \mathrm{~nm}$. Further, silicon oxide layers are formed on both sides of the ridge (sidewalls) surfaces of the silicon, and then the oxide layers are etched (Fig. 10 left). By repeating this process several times, it was possible to obtain extremely smooth surfaces with little scattering even in visible light. Specifically, a resist was formed on a silicon substrate by using a mask with a line \& space of $2.0 \mu \mathrm{m}: 3.1 \mu \mathrm{m}$. Deep grooves formed by the cycle etching onto the silicon substrates (two kind of depths with $10 \mu \mathrm{m}$ and $20 \mu \mathrm{m}$ ). Silicon oxide layers are formed and removed twice. Where oxygen diffuses from a surface of the silicon is about $400 \mathrm{~nm}$ by the oxidation process when an oxide layer having a thickness of about $1 \mu \mathrm{m}$. As a result, lattices of ridges with widths of 0.44 and 0.8 $\mu \mathrm{m}$, respectively, which surfaces are extremely smooth, are obtained (Fig. 10 middle and right).

\subsection{Quasi-Bragg immersion grating}

An immersion grating is a reflection grating filled with a high-refractive index medium in its optical path, and angular dispersion of the immersion grating is proportional to the refractive index of the medium $[24,25]$. In other words, since the size of an immersion grating can be reduced in inverse proportion to the refractive index of the medium compared with a conventional reflection grating with the same angular dispersion.

The QB immersion grating [11] was fabricated by combination of an inclined QB grating, Littrow prism and surface reflection mirror as shown on the right in figure 11 and on the left and middle in figure 12. The block of 60 seats of silica substrates was stacked by room temperature bonding of gold in Tohoku University (Fig. 11 left) [26]. The QB grating was cut with inclination of $30^{\circ}$ from the block of silica substrates (Fig. 11 middle). We assembled a spectroscopic optical system of the Littrow mount (angle between incident and diffracted beams is small), and observed a diffraction image of the QB immersion grating using a He-Ne laser as a light source. As a result, although the order could not be separated because the point source image of the pinhole was larger than the interval of the diffraction orders, the diffraction intensity was highly concentrated (blazed) as shown in the right of figure 12, we confirmed the QB immersion grating functions as an immersion grating. It is difficult to fabricate a QB immersion grating for visible to near infrared wavelength by lamination of mirror substrates. On the other hand, it is relatively easy to fabricate a QB immersion grating of silicon or germanium for mid and far infrared wavelength by the lamination of mirror substrates.
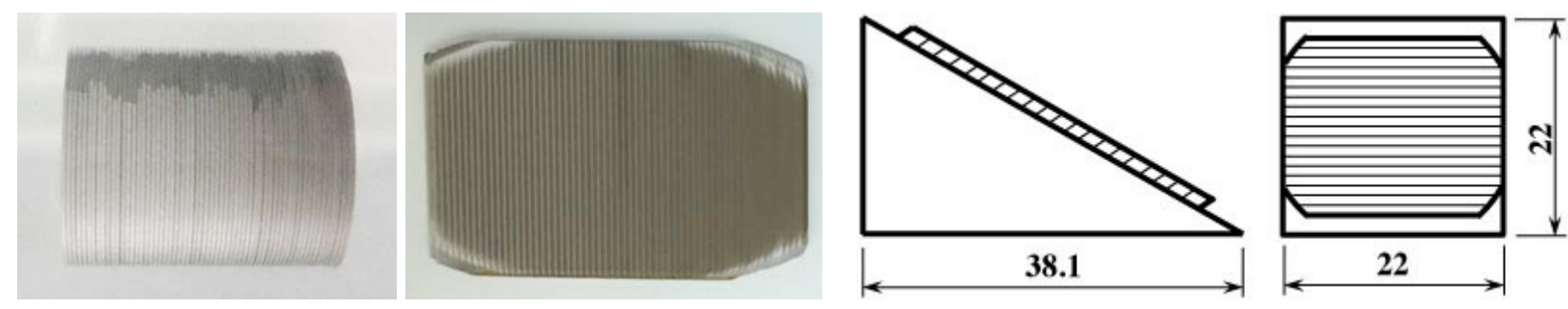

Figure 11 Block of silica substrate stacked by room temperature bonding of gold (left), inclined QB grating of QB immersion grating (middle) and schematic representation of prototype of QB immersion grating (right). 

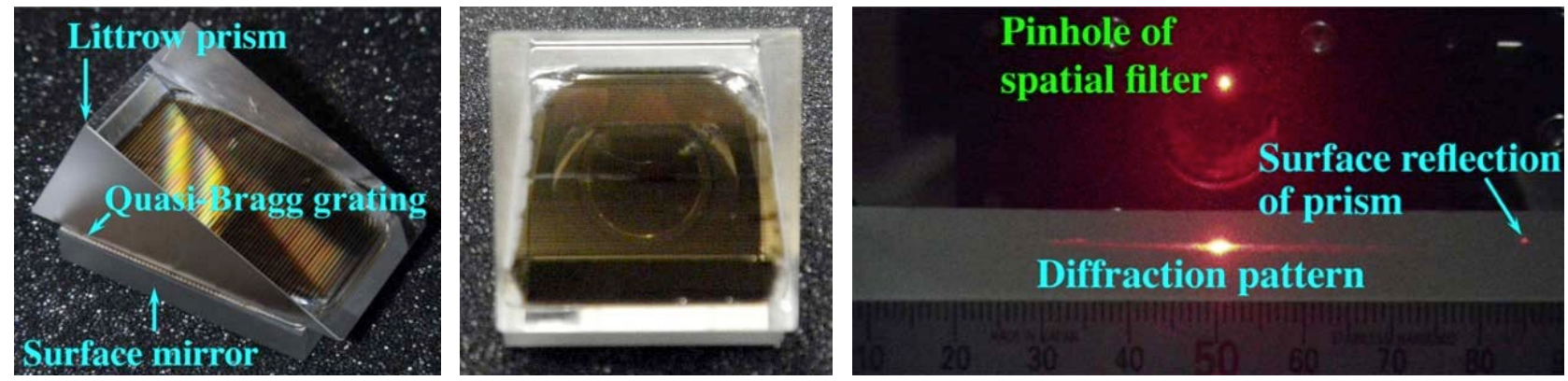

Figure 12 Prototype of QB immersion grating (left), camera lens image of diffraction beam gazed from square surface of Littrow prism (middle) and diffraction pattern of QB immersion grating (right).

\section{CONCLUSIONS}

The B-VPH grating introduced in this paper can achieve a maximum diffraction efficiency up to $100 \%$ at the 1 st diffraction order for natural polarization or circular polarization even when incident and diffraction angles (Bragg angle) exceeds $30^{\circ}$ in vacuum. We found that a B-VPH grating with high diffraction efficiency can be fabricated by using a commercially available UV curable LC and visible light curable resin. The RFT grating and the VB grating as a transmission grating can achieve high diffraction efficiency in higher diffraction orders. The mold of the SR transmission grating with an acute vertex angle for the hybrid grism as a prototype of a RFT grating was fabricated by shaper cutting process with our ultra-high precision machine and a single-crystal diamond bite. We succeeded in prototyping VB gratings of silicon as a mold with ridges of a high aspect ratio processed by cycle etching, oxidation of silicon and removal of the oxide layer. The QB immersion grating was fabricated by combination of a QB grating inclined at 30 degrees, Littrow prism and a surface reflection mirror. We confirmed the QB immersion grating functions as an immersion grating.

We appreciate Mr. Ken Kajiwara and Mr. Toshio Okumura of Nanotechnology Platform facilities, the Toyota Institute of Technology for their assistance on the trial fabrications of the silicon VB gratings. We utilize facility of the Advanced Technology Center of National Astronomical Observatory of Japan (NAOJ) for grating measurements and "Nanotechnology Platform Japan" of the Ministry of Education, Culture, Sports, Science and Technology (MEXT), Japan for grating fabrications. This work is supported by the grant-in-aid of NAOJ for TMT strategic basic research and development, by the Grant-in-Aid for Challenging Exploratory Research, 2015-2016, 15K13470; from MEXT. And this work was supported by the grant-in-aid of NAOJ for Joint development research, and by Adaptable and Seamless Technology Transfer Program through Target-driven R\&D from Japan Science and Technology Agency.

\section{REFERENCES}

[1] Takato, N. and Iwata, I., "Instrumentation at the Subaru Telescope," Proc. SPIE 8446, 8446051-0510 (2012).

[2] Ebizuka, N., et al., "Diffraction Gratings for the Latest Visible and Infrared Astronomical Observations," J. Jpn. Soc. Infrared Sci. \& Tech., 26, 32-39 (2017)

[3] Ebizuka, N., et al., "Cryogenic VPH Grisms for MOIRCS," PASJ 63, S605-S612 (2011).

[4] Ebizuka, N., et al., "Grisms Developed for FOCAS," PASJ 63, S613-S622 (2011).

[5] Uehara, M., et al., "Development of the Wide Field Grism Spectrograph 2," Proc. SPIE 5492, 661-668 (2004).

[6] Watanabe, M., et al., "TRISPEC: A Simultaneous Optical and Near-Infrared Imager, Spectrograph, and Polarimeter, ” PASP 117, 870-884 (2005).

[7] Ebizuka, N., et al., "Novel diffraction gratings for next generation Spectrographs with high spectral dispersion," Proc. SPIE 9912, 2Z1-2Z10 (2016)

[8] Oka, K., et al., "Optimization of a volume phase holographic grism for astronomical observation using the Photopolymer," Proc. SPIE 5005, 8-19 (2003).

[9] Barden, S.C., Arns; J.A. and Colburn, W.S., "Volume-phase holographic gratings and their potential for astronomical applications," Proc. SPIE 3355, 866-876 (1998). 
[10] Baldry, I. K., Bland-Hawthorn, J. and Robertson, J. G., "Volume Phase Holographic gratings: Polarization properties and Diffraction Efficiency," PASP 116, 403-414 (2004).

[11] Ebizuka, N., et al., "Birefringence Bragg Binary (3B) Grating, Quasi-Bragg Grating and Immersion Gratings," Proc. SPIE 9151, 5C1-5C9 (2014).

[12] Gupta, M. C. and Peng, S. T., "Diffraction characteristics of surface-relief gratings,” Appl. Opt. 32, 2911-2917 (1993).

[13] Gerritsen, H. J. and Jepsen, M. L., "Rectangular surface-relief transmission gratings with a very large first- order diffraction efficiency (95\%) for unpolarized light," Appl. Opt. 37, 5823-5829 (1998).

[14] Simard, N.L., et al., "The instrumentation program for the Thirty Meter Telescope," Proc. SPIE 8446, 1F1-1F10 (2012).

[15] Jacoby, G. H., et al., "The instrument development and selection process for the Giant Magellan Telescope," Proc. SPIE 8446, 1G1-1G16 (2012).

[16] Casali, M. M.; Pasquini, L. and Ramsay, S., “Overview of the ESO instrumentation program,” Proc. SPIE 8446, 041-047 (2012).

[17] Nakagawa, T., et al., "The next-generation infrared astronomy mission SPICA under the new framework," Proc. SPIE 9143, 1I1-1I9 (2014).

[18] Ichikawa, T., "MOIRCS: multi-object infrared camera and spectrograph for SUBARU," Proc. SPIE 6269, 16 (2006).

[19] Moharam, M. G. and Gaylord, T. K., "Rigorous coupled-wave analysis of planar-grating diffraction," JOSA 71, 811 (1981).

[20] Li, L., "Note on the S-matrix propagation algorithm," JOSA A 20, 655 (2003).

[21] Pazder, J. S., et al., "WFOS: a wide field optical spectrograph for the Thirty Meter Telescope," Proc. SPIE 6269, 1X (2006).

[22] Oka, K.; Ebizuka, N. and Kodate, K., "Optimal design of the grating with reflective plate of comb type for astronomical observation using RCWA,” Proc. SPIE 5290, 168-178 (2004).

[23] Bianco, A. and Ebizuka, N., "Echelle VPHG: a step forward,” Proc. SPIE 8450, 431-438 (2012).

[24] Larsson, T and Neuhaus, "The Immersion Grating: Spectroscopic Advantages and Resemblance to the Echelon Grating" Zeitschrift fr Naturforschung A 23, 2130-2032 (1968).

[25] Wiedemann, G. and Jennings, D.E., "Immersion grating for infrared astronomy," Appl. Opt. 32, 1176-1178 (1993)

[26] Shimatsu, T. and Uomoto, M., "Room Temperature Bonding of Wafers with Thin Nanocrystalline Metal Films," ECS Transactions 33, 61-72 (2010). 